\title{
THE "BRUNELLA" PROJECT: PREPARATION AND STUDY OF A MYSTICETE FROM THE EARLY PLIOCENE OF TUSCANY
}

\author{
Roberta Scotton ${ }^{1}$, Renzo Bigazzi ${ }^{1}$, Simone Casati², Giuseppe D’Amore ${ }^{1}$, Sylvia Di Marco ${ }^{1}$, \\ Luca Maria Foresi ${ }^{3}$, Elizabeth Koenig ${ }^{4}$, Luca Ragaini ${ }^{5}$, Jacopo Tabolli ${ }^{6}$, Massimo Tarantini ${ }^{7}$, \\ Giandonato Tartarelli ${ }^{8} \&$ Michelangelo Bisconti ${ }^{9}$
}

\begin{abstract}
${ }^{1}$ Istituto di Studi Archeo-Antropologici, Via delle Cascine 46, 50018, Scandicci (Firenze); robertascotton@yahoo.it; renzo.bigazzi@ teletu.it; g_damore@libero.it; sylvia_dimarco@libero.it

${ }^{2}$ Gruppo AVIS di Mineralogia e Paleontologia Scandicci, Piazza Vittorio Veneto 1, 50018, Scandicci (Firenze); simonecasati@alice.it ${ }^{3}$ Dipartimento di Scienze Fisiche, della Terra e dell'Ambiente, Università degli Studi di Siena, via Laterina 8, 53100, Siena; luca.foresi@ unisi.it

${ }^{4}$ Banfi s.r.l., Castello di Poggio alle Mura, 53024, Montalcino (Siena); elizabeth.koenig@banfi.it ${ }^{5}$ Dipartimento di Scienze della Terra, Università di Pisa, via Santa Maria 53, 56126, Pisa; ragaini@dst.unipi.it

${ }^{6}$ Soprintendenza Archeologia, Belle Arti e Paesaggio per le province di Siena, Grosseto, Arezzo, via di Città 138/140, 53100, Siena; jacopo. tabolli@beniculturali.it;

${ }^{7}$ Soprintendenza Archeologia, Belle Arti e Paesaggio per la città metropolitana di Firenze e le province di Pistoia e Prato, Piazza de' Pitti 1, 50125, Firenze; massimo.tarantini@beniculturali.it

${ }^{8}$ Scuola Normale Superiore, Piazza Cavalieri, 56100, Pisa; tartarelli@sns.it

${ }^{9}$ San Diego Natural History Museum, 1788 El Prado, CA 92101, San Diego, United States of America; michelangelobisconti@gmail. com
\end{abstract}

\section{KEYWORDS: \\ mysticeti; \\ Pliocene; \\ Tuscany; \\ anatomy; \\ paleoenvironment}

\section{Bullet-Points Abstract}

- A project is being carried out at Poggio alle Mura that will allow the preparation and study of a balaenopterid whale from the early Pliocene of Tuscany together with its associated biota.

- Up to now, the project resulted in the preparation of 18 vertebrae, the skull and an ulna of the whale.

- The associated biota includes 18 mollusk species, 2 sea urchin species, 10 shark teeth and thousands of fish remains from the sediment surrounding the skull.

- A wealth of educational activities is being carried out at the laboratory of Poggio alle Mura that are directed to schools and citizens.

\section{INTRODUCTION}

In 2007, the skeleton of a mysticete was uncovered in the Banfi's vineyard at the locality of Poggio alle Mura, near Montalcino (Siena). The excavation of this whale resulted in the discovery of a partially articulated skeleton including skull, both dentaries, part of the vertebral column, one scapula, parts of the forelimbs and ribs. Since the excavation this whale has been referred to with the nickname "Brunella". The excavation revealed also mollusks, barnacles and palaeobotanical remains. In addition, a preliminary investigation of the stratigraphy at the site was carried out and sediment samples for micropalaeontological analyses were gathered from the stratigraphic level where the whale was found and from different layers of the stratigraphic section. The excavation occurred during an important enological event in Montalcino and the discovery of the whale was presented on national television channels (RAI) and onto numerous national and international newspapers and journals (e.g., Anonymous, 2007). In the following years, the fossils resulting from the 2007 excavation were stored in a warehouse at the Banfi's property at Poggio alle Mura. In 2016, the Soprintendenza Archeologica della Toscana decided to begin a new project focused on the preparation, study and possible exhibition of this whale on the basis of early reports suggesting that it could be an important step towards shedding light on mysticete evolution, and on palaeobiogeographic and palaeoecological conditions at the site of the discovery.

\section{Materials AND Methods}

The mysticete specimen and the sediment samples taken at the discovery site are preserved in the laboratory at Poggio alle Mura (Montalcino, Siena Pro vince). The geological section at the site was studied during the excavation in 2007 and samples were obtai- 


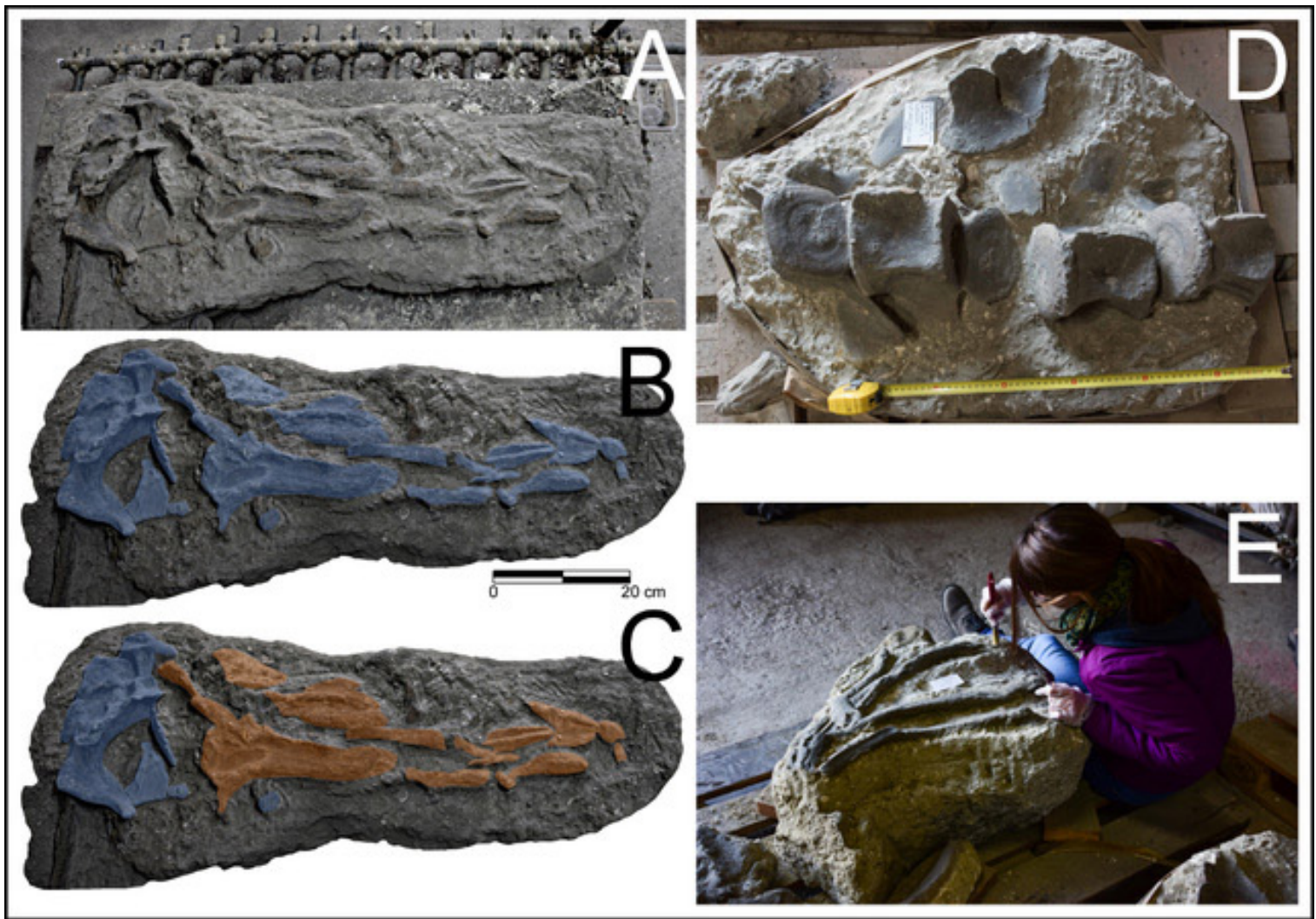

Fig. 1. The "Brunella" project. A, the skull of the mysticete in the slab where it was preserved together with abundant mollusks. $\mathbf{B}$, another view of the skull with the bones digitally colored in blue for closer individuation. $\mathbf{C}$, the skull with rostrum digitally colored in orange and neurocranium in blue. $\mathbf{D}$, a group of lumbar vertebrae, caudal vertebrae and chevrons in the slab where they are preserved. E, a student of a summer school during preparation activities on "Brunella"'s ribs.

ned from different layers to be used for micropalaeontological analyses. Sediments were washed and sieved to be examined under microscopy in search for microfossils. The mysticete bones were subject to mechanical preparation; use of alcohol and oxidized water supplemented the mechanical preparation to facilitate sediment removal. The sediment removed from the bones was washed and sieved to be analyzed under a stereomicroscope with magnification of $10 \mathrm{x}$ and $20 \mathrm{x}$. Standard sediment samples were 11 in volume corresponding to $1476 \mathrm{~g}$ in weight. All the microfossils were saved in dedicated containers. During the microexcavation of the bones, the chemical product used in 2007 to consolidate the skeleton was removed by application of acetone and a new bicomponent chemical product was then applied to definitively consolidate the bones. Mollusk shells were mechanically polished. The total body length of the whale was calculated by using dedicated regression equations (Pyenson et al., 2013) based on a preliminary estimation of the length of the right dentary.

\section{Discussions AND Conclusions}

The new project involved both public and private institutions in a joint effort with the following goals: prepare the specimen; obtain a chronostratigraphic assessment of its age; describe and analyze the palaeoenvironment at the site of the discovery based on mollusk, foraminifer and sediment analyses; describe the specimen and investigate into its systematic, phylogenetic and palaeobiogeographic relationships; exhibit the skeleton within an existent or new location (e.g., a museum); and, realize a series of educational programs around the specimen, such as a summer school focused on the preparation of paleontological remains open to undergraduate students. The project was funded by the Banfi Society following the recently established Art Bonus scheme of the MiBACT (Italian Ministry of Cultural Heritage and Tourism). To our knowledge, this is the first project of this kind focused on a fossil vertebrate to be developed in Italy.

The project started following separate agreements between the Archaeological Superintendency of Tuscany, the Banfi Society, and the Istituto di Studi Archeo-antropologici (ISA), resulting in two editions of a summer school realized in 2016 and 2017 involving teachers from three universities of Tuscany, the Scuola Normale Superiore of the University of Pisa, the ISA, the Museo di Storia Naturale di Milano, and the San Diego Natural History Museum (Fig. 1). The third edition of the summer school is currently under preparation and will take place by the end of 2018. The school has received the official endorsements by the 
Società Paleontologica Italiana (SPI) in previous editions, the Dipartimento di Scienze Fisiche, della Terra e dell'Ambiente of the University of Siena, and the Associazione Italiana per lo Studio del Quaternario (AIQUA). The following subjects have been included in the programs of the school: mysticete osteology, evolution and biogeography; general biological and geological patterns in the Mediterranean Pliocene; Miocene and Pliocene mollusk evolution; mysticete taphonomy; palaeogeographic and palaeoenvironmental evolution of Tuscany during the Pliocene; paleontological excavation techniques; techniques of preparation and restoration of fossil vertebrates; techniques of photogrammetry and tridimensional modelling of fossil vertebrates; and museology. Students from different universities came from northern, central and southern Italy sharing their cultural backgrounds: Geology, Natural Sciences and Conservation of Cultural Heritage. The Banfi Society provided the location for the classes, the warehouse for the preparation laboratory of the fossil whale (as well as food). RAI3 (national television channel), local newspapers and web journals provided media coverage on the different activities of the school. Presently (June 2018), the preparation and restoration of the specimen is in progress. The technicians are using mechanical preparation instruments to remove the matrix from the bones and a diverse array of chemical products to consolidate and preserve the fossil. The preservation is variable: the vertebrae and the posterior portion of the skull are in good conditions while the rostrum is badly damaged by post-mortem processes. The scientific study of "Brunella" is still in progress. Preliminary investigations revealed that: it belonged to the family Balaenopteridae; it does not belong to any of the living taxa; based on the length of its dentary, it was estimated that the total body length of "Brunella" should have been between c. 6 and c. 7.5 $\mathrm{m}$ and its weight was c. $5000 \mathrm{~kg}$; the age of this whale was preliminarily assessed based on foraminifers and mollusks and was constrained between 3.7 and 4.5 Ma. "Brunella" was framed in publications on the taphonomy and stratigraphic palaeobiology of baleen whales from Italy (Dominici et al., 2009; 2018). Given its degree of completeness and preservation, it is expected that "Brunella" will shed further light on the evolution of balaenopterid mysticetes from the Early Pliocene of the Mediterranean basin.

\section{ACKNOWLEDGEMENTS}

The "Brunella" project was made possible by the interest and action of several individuals: SC discovered the specimen together with Franco Gasparri; Menotti Mazzini (former technician of the University of Florence) directed the 2007 excavation; Enrico Viglierchio, Remo Grassi (all at Banfi s.r.1.), Giorgio Carnevale (University of Turin), Cristiano Dal Sasso (Natural History Museum of Milan) provided valuable insights and help during the research and the field school editions.

\section{REFERENCES}

Anonymous (2007). The whales of Italy. Science, 316: 179.

Dominici S., Cioppi E., Danise S., Betocchi U., Gallai G., Tangocci F., Valleri G. \& Monechi S. (2009). Mediterranean fossil whale falls and the adaptation of mollusks to extreme habitats. Geology, 37: 815-818.

Dominici S., Danise S. \& Benvenuti M. (2018). Pliocene stratigraphic paleobiology in Tuscany and the fossil record of marine megafauna. Earth Sciences Reviews, 176: 277-310.

Pyenson J. D., Goldbogen J. A., Shadwick R. E. (2013). Mandible allometry in extant and fossil Balaenopteridae (Cetacea: Mammalia); the largest vertebrate skeletal element and its role in rorqual lunge feeding. Biological Journal of the Linnean Society, 108: 586-599.

Manuscript received 13 July 2018

Received after revision 25 September 2018 Accepted 2 October 2018 